\title{
ISOMORPHIC COMPLEXES. II
}

\author{
BY JACK SEGAL
}

Communicated by O. G. Harrold, September 30, 1965

In a preceding note [2] we showed that if $K$ and $L$ are $n$-complexes, then $K$ and $L$ are isomorphic iff the 1-sections of the first derived complexes of $K$ and $L$ are isomorphic. Since topological equivalence does not imply combinatorial equivalence for complexes this result fails to hold if the 1-sections are only required to be homeomorphic. However, for a large class of complexes we will show that this theorem is true under the weaker condition.

Throughout, $s_{p}$ will denote a (rectilinear) $p$-simplex with vertices $a^{0}, a^{1}, \cdots, a^{p} ; K$ will denote a finite geometric simplicial complex with $n$-section $K^{n}$ and first derived complex $K^{\prime}$. For more details see $[1, \S 1.2]$.

We first recall a definition and two theorems from [2].

Definition 1. An $n$-complex $K$ is full provided, for any subcomplex $L$ of $K$ which is isomorphic to $s_{p}^{1}, 2 \leqq p \leqq n, L^{0}$ spans a $p$-simplex of $K$.

Theorem 1. If $K$ and $L$ are full $n$-complexes, then $K$ and $L$ are isomorphic iff $K^{1}$ and $L^{1}$ are isomorphic.

THeorem 2. If $K$ and $L$ are $n$-complexes, then $K$ and $L$ are isomorphic iff $\left(K^{\prime}\right)^{1}$ and $\left(L^{\prime}\right)^{1}$ are isomorphic.

Definition 2. A complex $K$ is said to be taut provided, $K^{1}$ has no vertex of order 2 .

Definition 3. A complex $K$ is said to be trim if it is full and taut.

In each of the next three theorems we need only prove one implication for the equivalence since isomorphic complexes have homeomorphic realizations.

Theorem 3. If $K$ and $L$ are taut 1-complexes, then $K$ and $L$ are isomorphic iff $|K|$ and $|L|$ are homeomorphic.

Proof. Let $\phi:|K| \rightarrow|L|$ be a homeomorphism of $|K|$ onto $|L|$. If $a$ is a vertex of $K$, then the order of $\phi(a)$ is not two since order is a topological property. So $\phi(a)$ is a vertex of $L$. Hence $L$ has at least as many vertices as $K$. Similarly, using $\phi^{-1}$ instead of $\phi$ we obtain that $K$ has at least as many vertices as $L$. So $K$ and $L$ have the same number of vertices. Therefore, $v: K \rightarrow L$ defined by

$$
v(a)=\phi(a), \quad a \in K^{0}
$$


is a vertex transformation of $K$ to $L$ taking $K^{0}$ onto $L^{0}$ in a 1-1 fashion.

We will now show that $v$ is admissible. If $a^{0}, a^{1}$ span a 1 -simplex of $K$, then $v\left(a^{0}\right), v\left(a^{1}\right)$ span a 1 -simplex of $L$ since $v\left(a^{0}\right), v\left(a^{1}\right) \in L^{0}$ are the end points of the $\operatorname{arc} \phi\left[\left|a^{0} a^{1}\right|\right]$ which contains no other vertices of $L$. So $v$ is admissible. A similar argument shows $v^{-1}$ is also an admissible vertex transformation. Hence $v$ induces an isomorphism of $K$ onto $L$.

Theorem 4. If $K$ and $L$ are trim $n$-complexes, then $K$ and $L$ are isomorphic iff $\left|K^{1}\right|$ and $\left|L^{1}\right|$ are homeomorphic.

Proof. Suppose $\left|K^{1}\right|$ and $\left|L^{1}\right|$ are homeomorphic. Then since they are taut, we have $K^{1}$ and $L^{1}$ are isomorphic by Theorem 3. Since $K$ and $L$ are full, Theorem 1 applies and so $K$ and $L$ are isomorphic.

Theorem 5. If $K^{\prime}$ and $L^{\prime}$ are taut $n$-complexes, then $K$ and $L$ are isomorphic iff $\left|\left(K^{\prime}\right)^{1}\right|$ and $\left|\left(L^{\prime}\right)^{1}\right|$ are homeomorphic.

Proof. Suppose $\left|\left(K^{\prime}\right)^{1}\right|$ and $\left|\left(L^{\prime}\right)^{1}\right|$ are homeomorphic. Then since they are taut we have $\left(K^{\prime}\right)^{1}$ and $\left(L^{\prime}\right)^{1}$ are isomorphic by Theorem 3. So Theorem 2 applies and we have that $K$ and $L$ are isomorphic.

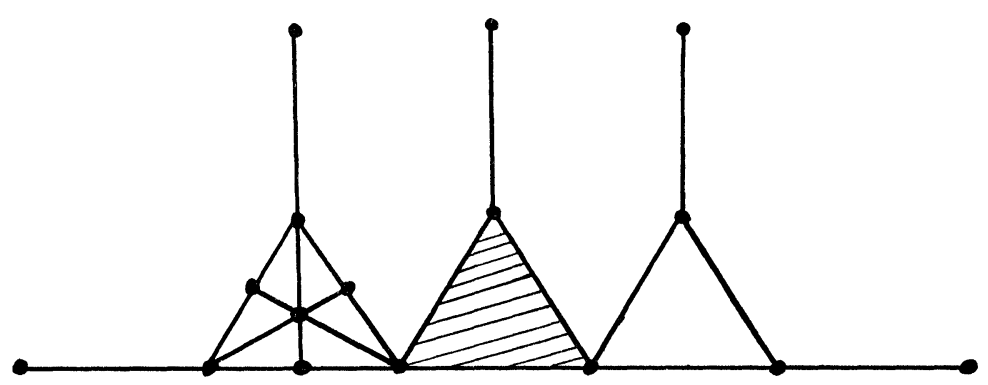

(K)

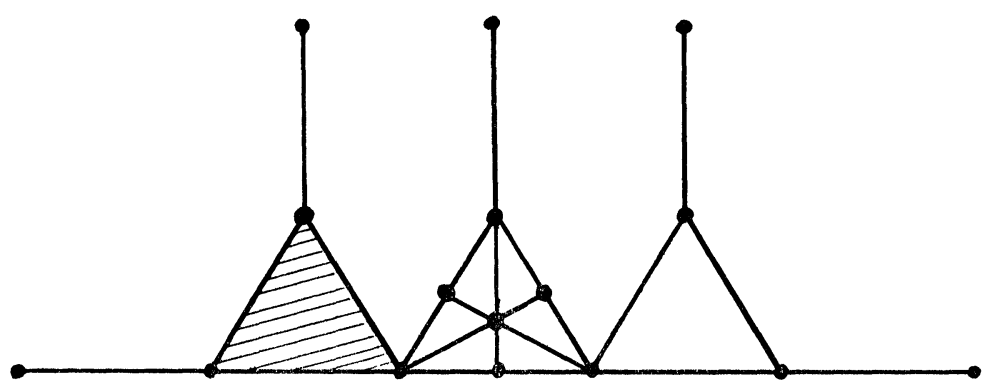

(L) 
Example 1. Let $K=\left(s_{2}^{1}\right)^{\prime}$ and $L=K^{\prime}$. Then $K$ and $L$ are nonisomorphic full 1-complexes and $\left|K^{1}\right|$ and $\left|L^{1}\right|$ are homeomorphic. This shows the need for requiring tautness in Theorems 4 and 5.

Example 2. That tautness of $K$ and $L$ is not a strong enough requirement in Theorem 5 is shown by the preceding example of two taut nonisomorphic 2-complexes with $\left|\left(K^{\prime}\right)^{1}\right|$ and $\left|\left(L^{\prime}\right)^{1}\right|$ being homeomorphic.

\section{REFERENCES}

1. P. J. Hilton and S. Wiley, Homology theory, Cambridge Univ. Press, New York, 1960.

2. J. Segal, Isomorphic complexes, Bull. Amer. Math. Soc. 71 (1965), 571-572.

UNIVERSITY OF WASHINGTON 